\title{
Interplay of quark and meson degrees of freedom in a near-threshold resonance
}

\author{
V. Baru ${ }^{1,2}$, C. Hanhart ${ }^{2}$, Yu. S. Kalashnikova ${ }^{1}$, A. E. Kudryavtsev ${ }^{1}$, A. V. Nefediev ${ }^{1}$ \\ 1 Institute for Theoretical and Experimental Physics, 117218, B. Cheremushkinskaya 25, Moscow, Russia \\ 2 Forschungszentrum Jülich, Institut für Kernphysik (Theorie) and Jülich Center for Hadron Physics, D-52425 Jülich, Germany
}

\begin{abstract}
We investigate the interplay of quark and meson degrees of freedom in a physical state representing a near-threshold resonance for the case of a single continuum channel. We demonstrate that such a near-threshold resonance may possess quite peculiar properties if both quark and meson dynamics generate weakly coupled near-threshold poles in the S-matrix. In particular, the scattering t-matrix may possess zeros in this case. We also discuss possible implications for production reactions as well as studies within lattice QCD.
\end{abstract}

PACS. 12.38. $\mathrm{Lg}-11.55 . \mathrm{Bq}-12.39 . \mathrm{Mk}$

\section{Introduction}

With recent developments of $B$-factories, a wealth of new charmonia states was reported, with properties of some of those being incompatible with simple quark model predictions. Exotic explanations, such as hybrids or tetraquarks, are suggested to describe the new states. On the other hand, these new enigmatic states are above open charm threshold, so that the spectrum of the "quenched" quark model is to be significantly distorted by charmed-meson pairs (for reviews, see e.g. Refs. [1,2, 3, 4, 5]).

Moreover, some of the new states reside in the vicinity of $S$-wave thresholds. A most prominent example here is the famous $X(3872)$ state, first observed in $B$-meson decays [6]. The $X(3872)$ is extremely close to the $D \bar{D}^{*}$ threshold, with $D \bar{D}^{*}$ being in the $S$-wave, if the quantum numbers of the $X$ are $1^{++}$, as suggested by the data. There are also the $Y(4260)$ and $Y(4325)$ vector states [7,8, with relevant $S$-wave thresholds being the $D \bar{D}_{1}$ at $4.285 \mathrm{GeV}$ and $D^{*} \bar{D}_{0}$ at about $4.360 \mathrm{GeV}\left(D_{1}\right.$ and $D_{0}$ are correspondingly $1^{+}$and $0^{+} D$-mesons $)$. The purely exotic charged state $Z^{+}(4430)$ [9 is close to the $D^{*} \bar{D}_{1}$ threshold and, for the charged $Z_{1}^{+}(4050)$ and $Z_{2}^{+}(4250)$ [10, the relevant thresholds are $D^{*} \bar{D}^{*}$ and $D_{1} \bar{D}$, respectively 1 .

Threshold proximity implies that, independently of the binding mechanism, there should be a significant component of a hadronic molecule in the wave function of the state. So the question arises of how to distinguish between a genuine "elementary" particle ( $q \bar{q}$, hybrid, or compact

\footnotetext{
1 These charged states are to be considered with caution, as seen only in one experiment (Belle). Besides, as quantum numbers of the $Z$-particles are not known, the thresholds are not necessarily the $S$-wave ones.
}

tetraquark) and a composite state (hadronic molecule), and how to estimate the admixture of the latter. It was suggested in Ref. 11, 12,13, that it is possible, in the case of a near-threshold bound state, to answer this question in a model-independent way: the state is mostly elementary if the effective radius is large and negative. The approach was generalised in Ref. 14,15 to the case of presence of inelastic channels, as well as to the case of an abovethreshold resonance. Related to this is the pole counting approach [16], in which the structure of the near-threshold singularities of the scattering amplitude is studied. It appears that the state is mostly elementary if there are two nearby poles in the scattering amplitude, while a composite particle corresponds to a single near-threshold pole.

The approaches of Ref. [11, 12, 13, 14, 15, 16, are based on the effective-range expansion of the scattering amplitude and, as such, are expected to be valid for the momenta involved much smaller than the inverse range of the force. However, as noticed in Ref. 11, 12, 13, the effectiverange formulae are not the most general ones even in the small-momenta limit. The scattering amplitude, as a function of energy, can have a zero and, if this zero is situated in the near-threshold region, the effective-range expansion would fail. In the present paper we develop a formalism which allows one to pinpoint the source of this failure, to study the hadronic observables in the presence of this zero, and to identify the physical situation in which it occurs.

\section{General formalism}

We consider a physical state which is a mixture of a bare state ( $q \bar{q}$ or compact tetraquark) and a dynamical (mole- 
cule) component and represent its wave function as:

$$
|\Psi\rangle=\left(\begin{array}{c}
c\left|\psi_{0}\right\rangle \\
\chi\left|M_{1} M_{2}\right\rangle
\end{array}\right)
$$

where $\left|\psi_{0}\right\rangle$ is the bare elementary state with the probability amplitude $c$, while $\chi(\boldsymbol{p})$ describes the relative motion in the system of two mesons $\left(M_{1} M_{2}\right)$, with the masses $m_{1}$ and $m_{2}$, respectively, and with the relative momentum $\boldsymbol{p}$. The wave function $|\Psi\rangle$ obeys a Schrödinger-like equation:

$$
\mathcal{H}|\Psi\rangle=E|\Psi\rangle,
$$

with the Hamiltonian

$$
\mathcal{H}=\left(\begin{array}{cc}
H_{0} & V_{q h} \\
V_{h q} & H_{h}
\end{array}\right),
$$

where

$$
H_{0}\left|\psi_{0}\right\rangle=E_{0}\left|\psi_{0}\right\rangle,
$$

$m_{1}+m_{2}+E_{0}$ is the bare state mass, and

$$
H_{h}\left(\boldsymbol{p}, \boldsymbol{p}^{\prime}\right)=\frac{p^{2}}{2 \mu} \delta\left(\boldsymbol{p}-\boldsymbol{p}^{\prime}\right)+V\left(\boldsymbol{p}, \boldsymbol{p}^{\prime}\right),
$$

where $\mu$ is the reduced mass.

The term $V_{q h}$ is responsible for the dressing of the bare state, which is given by the transition form factor $f(\boldsymbol{p})$ :

$$
\left\langle\psi_{0}\left|V_{q h}\right| M_{1} M_{2}\right\rangle=f(\boldsymbol{p}) .
$$

The Schrödinger-like equation (2) is equivalent to the system of equations for the $c$ and $\chi$, which reads:

$$
\left\{\begin{array}{l}
c(E) E_{0}+\int f(\boldsymbol{p}) \chi(\boldsymbol{p}) d^{3} p=c(E) E, \\
\frac{p^{2}}{2 \mu} \chi(\boldsymbol{p})+c(E) f(\boldsymbol{p})+\int V(\boldsymbol{p}, \boldsymbol{k}) \chi(\boldsymbol{k}) d^{3} k=E \chi(\boldsymbol{p}) .
\end{array}\right.
$$

On substituting $c(E)$ from the first equation to the second one, we arrive at the Schrödinger equation in the mesonic channel, with an effective potential

$$
V_{\mathrm{eff}}\left(\boldsymbol{p}, \boldsymbol{p}^{\prime}, E\right)=V\left(\boldsymbol{p}, \boldsymbol{p}^{\prime}\right)+\frac{f(\boldsymbol{p}) f\left(\boldsymbol{p}^{\prime}\right)}{E-E_{0}} .
$$

The off-shell mesonic $t$-matrix $t\left(\boldsymbol{p}, \boldsymbol{p}^{\prime}, E\right)$ is a solution of the Lippmann-Schwinger equation,

$t\left(\boldsymbol{p}, \boldsymbol{p}^{\prime}, E\right)=V_{\mathrm{eff}}\left(\boldsymbol{p}, \boldsymbol{p}^{\prime}, E\right)-\int d^{3} q \frac{V_{\mathrm{eff}}(\boldsymbol{p}, \boldsymbol{q}, E) t\left(\boldsymbol{q}, \boldsymbol{p}^{\prime}, E\right)}{q^{2} /(2 \mu)-E-i 0}$.

The solution of this equation can be written as

$$
t\left(\boldsymbol{p}, \boldsymbol{p}^{\prime}, E\right)=t_{V}\left(\boldsymbol{p}, \boldsymbol{p}^{\prime}, E\right)+\frac{\phi(\boldsymbol{p}, E) \bar{\phi}\left(\boldsymbol{p}^{\prime}, E\right)}{E-E_{0}+\mathcal{G}(E)},
$$

\footnotetext{
${ }^{2}$ Normalisation of the $t$-matrix is such that the $M_{1} M_{2}$ scattering amplitude is given by $f(\boldsymbol{k}, \boldsymbol{k}, E)=-4 \pi^{2} \mu t(\boldsymbol{k}, \boldsymbol{k}, E)$, with $E=k^{2} /(2 \mu)$.
}

where $t_{V}\left(\boldsymbol{p}, \boldsymbol{p}^{\prime}, E\right)$ is the $t$-matrix for the potential problem,

$$
t_{V}\left(\boldsymbol{p}, \boldsymbol{p}^{\prime}, E\right)=V\left(\boldsymbol{p}, \boldsymbol{p}^{\prime}\right)-\int d^{3} q \frac{V(\boldsymbol{p}, \boldsymbol{q}) t_{V}\left(\boldsymbol{q}, \boldsymbol{p}^{\prime}, E\right)}{q^{2} /(2 \mu)-E-i 0},
$$

while the dressed vertex functions are

$$
\begin{aligned}
& \phi(\boldsymbol{p}, E)=f(\boldsymbol{p})-\int d^{3} q \frac{t_{V}(\boldsymbol{p}, \boldsymbol{q}, E) f(\boldsymbol{q})}{q^{2} /(2 \mu)-E-i 0}, \\
& \bar{\phi}(\boldsymbol{p}, E)=f(\boldsymbol{p})-\int d^{3} q \frac{t_{V}(\boldsymbol{q}, \boldsymbol{p}, E) f(\boldsymbol{q})}{q^{2} /(2 \mu)-E-i 0},
\end{aligned}
$$

and

$$
\mathcal{G}(E)=\int d^{3} q \frac{f(\boldsymbol{q}) \phi(\boldsymbol{q}, E)}{q^{2} /(2 \mu)-E-i 0} .
$$

The system of equations (7) can possess bound states (generally, more than one). For a bound state $i$ with the binding energy $\epsilon_{B}^{(i)}$, the solution of the system (7) may be written as

$$
c_{B}^{(i)}=\cos \theta_{i}, \quad \chi_{B}^{(i)}(\boldsymbol{p})=\psi_{i}(\boldsymbol{p}) \sin \theta_{i},
$$

where $\psi_{i}(\boldsymbol{p})$ is normalised to unity. Then the wave function of this bound state,

$$
|\Psi\rangle_{B}^{(i)}=\left(\begin{array}{c}
\cos \theta_{i}\left|\psi_{0}\right\rangle \\
\sin \theta_{i} \psi_{i}(\boldsymbol{p})\left|M_{1} M_{2}\right\rangle
\end{array}\right),
$$

is also normalised, ${ }_{B}^{(i)}\langle\Psi \mid \Psi\rangle_{B}^{(i)}=1$.

The quantity

$$
Z_{i}=\left|\left\langle\psi_{0} \mid \Psi\right\rangle_{B}^{(i)}\right|^{2}=\cos ^{2} \theta_{i},
$$

introduced in Ref. [11,12,13], gives the probability to find a bare state in the wave function of the bound state $i$.

The solution of the system (7) for the continuum is

$$
\begin{gathered}
c_{k}(E)=\frac{1}{E-E_{0}} \int d^{3} p \chi_{k}(\boldsymbol{p}) f(\boldsymbol{p})=\frac{\bar{\phi}(\boldsymbol{k}, E)}{E-E_{0}+\mathcal{G}(E)}, \\
\chi_{k}(\boldsymbol{p})=\delta(\boldsymbol{p}-\boldsymbol{k})-\frac{t(\boldsymbol{p}, \boldsymbol{k}, E)}{p^{2} /(2 \mu)-E-i 0}, \quad E=\frac{k^{2}}{2 \mu},
\end{gathered}
$$

so that the continuum counterpart of the quantity (17), the spectral density $w(E)$, gives the probability to find the bare state in the continuum wave function [17. It can be found as:

$$
w(E)=4 \pi \mu k\left|c_{k}(E)\right|^{2} \Theta(E),
$$

with $c_{k}(E)$ given by Eq. (18). As shown in Ref. [17, the normalisation condition for the distribution $w(E)$ reads:

$$
\int_{0}^{\infty} w(E) d E=1-\sum_{i} Z_{i},
$$

where the sum goes over all bound states present in the system, while the corresponding $Z$-factors are given by Eq. (17).

We are interested in the energy range close to the threshold. To perform a low-energy reduction, let us assume that the scattering length approximation is valid for 
the potential problem, so that the potential $t$-matrix $t_{V}$ takes the form:

$$
t_{V}(\boldsymbol{q}, \boldsymbol{p}, E)=-\frac{1}{4 \pi^{2} \mu\left(-a_{V}^{-1}-i k\right)}+\ldots,
$$

where $a_{V}$ is the scattering length for the potential $V\left(\boldsymbol{p}, \boldsymbol{p}^{\prime}\right)$ and ellipsis stands for the range of forces corrections. We use the sign convention, where a negative scattering length corresponds to an attractive potential, however, too weak to produce a bound state while a positive scattering length reverses to either a repulsive potential (which cannot produce a singularity of the $S$-matrix, thus we automatically have $1 / a_{V} \sim \beta, \beta$ is the range of forces) or a bound state, which is the case of interest here. Note, the above expression is useful only for $a_{V} \beta \gg 1$ and then applicable only for momenta of order $1 / a_{V}$. In this case $t_{V}$ has a nearthreshold pole. However, it should be stressed that this singularity is observable only in the weak coupling limit to the quark states located nearby, for otherwise all singularities undergo a significant mixing, the origin of which is the appearance of $t_{V}$ in the dressed vertex functions of Eqs. (12) and (13). For a detailed discussion of this effect see Ref. 18, 19 .

It is convenient to define the loop functions:

$$
\begin{aligned}
g(E) & =\int d^{3} q \frac{f^{2}(\boldsymbol{q})}{q^{2} /(2 \mu)-E-i 0}=f_{0}^{2}\left(R+4 \pi^{2} \mu i k\right), \\
g^{\prime}(E) & =\int d^{3} q \frac{f(\boldsymbol{q})}{q^{2} /(2 \mu)-E-i 0}=f_{0}\left(R^{\prime}+4 \pi^{2} \mu i k\right),
\end{aligned}
$$

where the constants $R$ and $R^{\prime}$ are expected to be of order $\mu \beta$ and corrections $O\left(k^{2} / \beta^{2}\right)$ where dropped; in addition we introduced $f_{0} \equiv f(0)$. Defining

$$
R_{V}=\frac{4 \pi^{2} \mu}{a_{V}}
$$

one arrives at the following form for the on-shell $t$-matrix (10):

$$
\begin{gathered}
t(E)=\frac{E-E_{C}}{\left[E-E_{0}\right] R_{V}+f_{0}^{2}\left[R R_{V}-R^{\prime 2}\right]+4 \pi^{2} \mu i k\left[E-E_{C}\right]}, \\
E_{C}=E_{0}-f_{0}^{2}\left(R+R_{V}-2 R^{\prime}\right) .
\end{gathered}
$$

Expression (25) for the scattering amplitude has a zero at $E=E_{C}$, and the effective-range expansion for the amplitude (25) is not valid, if $E_{C}$ is in the near-threshold region.

\section{Flatté formulae}

In this chapter we develop a generalisation of the wellknown Flatté parameterisation [20] for the near-threshold scattering amplitude, and express the $t$-matrix of Eq. (25) in terms of Flatté-type parameters.

Let us define the Flatté energy $E_{f}$ such that the real part of the denominator in Eq. (25) has a zero when $E=$ $E_{f}$,

$$
\left[E_{f}-E_{0}\right] R_{V}+f_{0}^{2}\left[R R_{V}-R^{\prime 2}\right]=0
$$

and get rid of $E_{0}$ this way. Then one can express $E_{C}$ in terms of $E_{f}$ :

$$
E_{C}=E_{f}-\frac{f_{0}^{2}}{R_{V}}\left(R^{\prime}-R_{V}\right)^{2},
$$

and, using the identity

$$
\frac{E-E_{f}}{E-E_{C}}=\frac{E-E_{f}}{E_{f}-E_{C}}-\frac{\left(E-E_{f}\right)^{2}}{\left(E-E_{C}\right)\left(E_{f}-E_{C}\right)},
$$

rewrite the $t$-matrix in the Flatté-type form as:

$$
t(E)=\frac{g_{f}}{8 \pi^{2} \mu \mathcal{D}_{F}(E)} .
$$

Here

$$
\frac{g_{f}}{2}=4 \pi^{2} \mu \frac{E_{f}-E_{C}}{R_{V}}=\frac{4 \pi^{2} \mu f_{0}^{2}}{R_{V}^{2}}\left(R-R_{V}\right)^{2}
$$

and

$$
\mathcal{D}_{F}(E)=E-E_{f}-\frac{\left(E-E_{f}\right)^{2}}{E-E_{C}}+\frac{i}{2} g_{f} k .
$$

If $\left|E_{C}\right| \gg\left|E_{f}\right|$, we are back to the standard Flatté approximation for the near-threshold scattering amplitude [20],

$$
t_{F}(E)=\frac{1}{8 \pi^{2} \mu} \frac{g_{f}}{E-E_{f}+\frac{i}{2} g_{f} k},
$$

which depends on two parameters, $g_{f}$ and $E_{f}$, in contrast to the expression (29), which depends on three parameters: $g_{f}, E_{f}$, and $E_{C}$. One can see that, for $\left|E_{C}\right| \sim\left|E_{f}\right|$, the standard Flatté formula is severely distorted by the presence of the $t$-matrix zero, with a drastic effect on the behaviour of the elastic scattering cross section.

The Flatté-type formulae given above define the $t$ matrix in terms of the parameters $E_{f}, g_{f}$, and $E_{C}$. Following S. Weinberg [11,12,13, it is instructive to consider the case of a single bound state present, and to express the $t$-matrix in terms of the binding energy $\epsilon_{B}$, the $Z$-factor (which is the probability to find a bare state in the physical bound state wave function), and the scattering length $a_{V}$. (25),

We start from the expression for the inverse $t$-matrix

$$
t^{-1}(E)=\frac{E-E_{f}}{E-E_{C}} R_{V}+4 \pi^{2} \mu i k
$$

In the vicinity of the bound-state pole one has

$$
t^{-1}(E) \simeq \frac{\epsilon_{B}+E}{g_{\mathrm{eff}}^{2}},
$$

with (see Ref. 11, 12, 13])

$$
g_{\mathrm{eff}}^{2}=\frac{\sqrt{2 \mu \varepsilon_{B}}}{4 \pi^{2} \mu^{2}}(1-Z) .
$$

This defines

$$
\frac{Z}{1-Z}=\frac{2 \epsilon_{B}}{\epsilon_{B}+E_{C}}\left(1-\frac{\gamma_{V}}{\sqrt{2 \mu \varepsilon_{B}}}\right),
$$


with $\gamma_{V}=1 / a_{V}$ being the inverse scattering length for the potential problem in the absence of the quark state. Thus the following formulae can be obtained [11,12,13]:

$$
k \cot \delta=-\sqrt{2 \mu \varepsilon_{B}}+\frac{\sqrt{2 \mu \varepsilon_{B}}\left(E+\epsilon_{B}\right)\left(\epsilon_{B}+E_{C}\right)}{2 \epsilon_{B}\left(E-E_{C}\right)} \frac{Z}{1-Z},
$$

and

$$
E_{C}=-\epsilon_{B}\left(1-\frac{2(1-Z)}{Z}+\frac{2(1-Z)}{Z} \frac{\gamma_{V}}{\sqrt{2 \mu \varepsilon_{B}}}\right) .
$$

Now, for $\left|E_{C}\right| \gg \epsilon_{B}$, the effective-range expansion is recovered from Eq. (37), with the scattering length $a$ and the effective radius $r_{e}$ given by:

$$
a=\frac{2(1-Z)}{(2-Z)} \frac{1}{\sqrt{2 \mu \varepsilon_{B}}}, \quad r_{e}=-\frac{Z}{(1-Z)} \frac{1}{\sqrt{2 \mu \varepsilon_{B}}},
$$

which allows one to define the quantity $Z$ in a modelindependent way, if the effective-range parameters $a$ and $r_{e}$ are available.

On the contrary, for $\left|E_{C}\right| \lesssim \epsilon_{B}$ the effective-range expansion does not converge anymore for energies larger than $\left|E_{C}\right|$. Correspondingly, formulae from Eq. (39) do not hold anymore. In the next section we discuss the circumstances, when the described unusual behaviour may occur.

\section{Pole structure in the presence of the $t$-matrix zero}

Due to a very simple relation (30) between $E_{C}$ and $\gamma_{V}$, it is instructive to study the amplitude singularities in terms of the variables $E_{f}, g_{f}$, and $\gamma_{V}$. The expression for the scattering t-matrix in this case takes the form:

$$
t(E)=\frac{1}{4 \pi^{2} \mu} \frac{E-E_{f}+\frac{1}{2} g_{f} \gamma_{V}}{\left(E-E_{f}\right)\left(\gamma_{V}+i k\right)+\frac{i}{2} g_{f} \gamma_{V} k} .
$$

As seen from expression (40), there generally could be up to three near-threshold poles, and the equation defining the pole positions in the $k$-plane reads:

$$
\left(k^{2}-2 \mu E_{f}\right)\left(\gamma_{V}+i k\right)+i \mu g_{f} \gamma_{V} k=0 .
$$

Denote the solutions of Eq. (41) as $k_{1}, k_{2}$, and $k_{3}$. As follows from the explicit form of Eq. (41), one of these solutions is always imaginary, while the other two are either both imaginary or placed at the lower half-plane symmetrically with respect to the imaginary axis, as required by the general properties of the $S$-matrix. Thus, the set of parameters $\left\{E_{f}, g_{f}, \gamma_{V}\right\}$ used in Eq. (40) is fully defined by the poles of the scattering $t$-matrix $\left\{k_{1}, k_{2}, k_{3}\right\}$ :

$$
\begin{aligned}
E_{f} & =-\frac{1}{2 \mu} \frac{k_{1} k_{2} k_{3}}{k_{1}+k_{2}+k_{3}}, \\
g_{f} & =-\frac{\left(k_{1}+k_{2}\right)\left(k_{1}+k_{3}\right)\left(k_{2}+k_{3}\right)}{i \mu\left(k_{1}+k_{2}+k_{3}\right)^{2}}, \\
\gamma_{V} & =-i\left(k_{1}+k_{2}+k_{3}\right) .
\end{aligned}
$$

The position $E_{C}$ of the $t$-matrix zero can be also expressed in terms of $\left\{k_{1}, k_{2}, k_{3}\right\}$ :

$$
E_{C}=E_{f}-\frac{1}{2} g_{f} \gamma_{V}=-\frac{1}{2 \mu}\left(k_{1} k_{2}+k_{1} k_{3}+k_{2} k_{3}\right) .
$$

Notice that, for the given $E_{f}$ and $\gamma_{V}$, the range of values for $E_{C}$ is restricted by the condition $g_{f} \geq 0$ (see Eq. (30) ), which follows from causality.

It is illustrating to discuss the behaviour of the three poles when the effective coupling $g_{f}$ is varied from zero to some large value. To study the properties of the system we introduce the scale $\Delta \ll \beta$ that defines the region of applicability of the equation 3 . If the coupling of the bare state to the mesonic channel is switched off, $g_{f}=0$, the solutions of Eq. (41) are

$$
k_{1,2}^{(0)}= \pm \sqrt{2 \mu E_{f}}, \quad k_{3}^{(0)}=i \gamma_{V} .
$$

The first pair of poles corresponds to the bare quark state (uncoupled from the mesonic channel) with the energy $E_{f}=E_{0}$, and the third one is due to the direct interaction $V\left(\boldsymbol{p}, \boldsymbol{p}^{\prime}\right)$ in the mesonic channel, as already described above. Note, however, that the numerator of the $t$-matrix (see Eq. (40)) has a zero exactly at the momenta $k_{1,2}^{(0)}$ which eliminates the contribution of the corresponding poles. Thus, in the limit of an extremely small coupling, $g_{f} \ll\left|E_{f}\right| /\left|\gamma_{V}\right|$ (see Eq. (45)), there is only one pole $k_{3}$ in the scattering $t$-matrix that corresponds to a bound or virtual state in the mesonic channel, depending on the sign of $\gamma_{V}$. As the interaction $g_{f}$ is switched on and increases, $g_{f} \sim\left|E_{f}\right| /\left|\gamma_{V}\right|$, the poles start to move and to mix, and the $t$-matrix may have a zero and three poles in different places of the $k$-plane. Note also that in this case there is no straightforward interpretation of $\gamma_{V}$ possible anymore due to the mixing of poles. Clearly, the zero of the $t$-matrix can have an impact on the observables only if it is in the region of applicability defined as $\Delta$. From Eq. (45) one can see then that $E_{C} \sim \Delta$ only if all three poles are located very close to the threshold. In other words, if either the potential pole or the quark poles are outside the region of applicability of our formalism, also the $t$-matrix zero lies outside that region. In the latter case the effective-range approximation is adequate for the problem under consideration, so that one can set $E_{C} \gg E, \Delta$ in all equations, thus recovering the standard Flatté formulae.

Let us now assume that all three bare poles appear in the vicinity of the threshold $\left(\left|E_{f}\right| \simeq \gamma_{V}^{2} /(2 \mu) \lesssim \Delta\right)$ but the coupling is strong $\left(g_{f} \gg\left|E_{f}\right| /\left|\gamma_{V}\right|\right)$. Clearly, in this case $E_{C} \gg \Delta$ and we again recover the standard lineshapes together with the standard effective range expansion; only a single pole remains in the near-threshold regime - the expressions of Eq. (39) hold again and we find, as expected, that this single pole corresponds to a state of predominantly dynamical (=molecular) origin. if:

To summarise, the most peculiar situation takes place

\footnotetext{
3 Note, however, that this applicability region might be further restricted by additional scales present in a particular problem under consideration.
} 
1. the bare quark and potential poles appear accidentally close to each other and to the threshold $\left(\left|E_{f}\right| \simeq\right.$ $\left.\gamma_{V}^{2} /(2 \mu) \lesssim \Delta\right)$

2. the quark state is relatively weakly coupled to the hadronic channel $\left(g_{f} \sim\left|E_{f}\right| /\left|\gamma_{V}\right|\right)$.

Thus, the appearance of a zero in the scattering amplitude very close to threshold, signaled also by an early breakdown of the effective-range expansion, is a clear signal of the presence of both potential as well as quark poles.

To detail these statements and to mimic the peculiar situation described above we choose $\left|\gamma_{V}\right| \sim \sqrt{2 \mu\left|E_{f}\right|}$ for $g_{f}=0$. We choose for the reduced mass $\mu=1 \mathrm{GeV}$ as is appropriate for the $X(3872)$ case, and define the nearthreshold region by $\Delta \sim 1 \mathrm{MeV}$ - the energy range relevant for the $X(3872)$ particle (in case of the $X(3872)$ the range of validity of the approach is not set by the range of forces, but by the closet threshold, which is only $8 \mathrm{MeV}$ away). We then study the system for different values of $g_{f}$ for six representative scenarios (recall: $\gamma_{V}<0$ refers to an attractive potential, which does not support a bound state, while $\gamma_{V}>0$ refers to a bound state present from the pure potential scattering). For each case we fix the parameters $E_{f}$ and $\gamma_{V}$, and change $g_{f}$ from very small values to the ones for which the $t$-matrix zero leaves the near-threshold region.

- Case (i): $E_{f}>0, \gamma_{V}>0\left(E_{f}=1 \mathrm{MeV}, \gamma_{V}=\right.$ $45 \mathrm{MeV}$ ). See Fig. 1, 2 ,

- Case (ii): $E_{f}>0, \gamma_{V}<0\left(E_{f}=1 \mathrm{MeV}, \gamma_{V}=\right.$ $-45 \mathrm{MeV})$. See Fig. 3 .

- Case (iii-a): $E_{f}<0, \gamma_{V}>0, \sqrt{2 \mu\left|E_{f}\right|}>\gamma_{V}\left(E_{f}=\right.$ $-1 \mathrm{MeV}, \gamma_{V}=40 \mathrm{MeV}$ ). See Fig. 4 .

- Case (iii-b): $E_{f}<0, \gamma_{V}>0, \sqrt{2 \mu\left|E_{f}\right|}<\gamma_{V}\left(E_{f}=\right.$ $-1 \mathrm{MeV}, \gamma_{V}=50 \mathrm{MeV}$ ). See Fig. 5 .

- Case (iv-a): $E_{f}<0, \gamma_{V}<0, \sqrt{2 \mu\left|E_{f}\right|}>\left|\gamma_{V}\right|\left(E_{f}=\right.$ $\left.-1 \mathrm{MeV}, \gamma_{V}=-20 \mathrm{MeV}\right)$. See Fig. 6.

- Case (iv-b): $E_{f}<0, \gamma_{V}<0, \sqrt{2 \mu\left|E_{f}\right|}<\left|\gamma_{V}\right|\left(E_{f}=\right.$ $-1 \mathrm{MeV}, \gamma_{V}=-55 \mathrm{MeV}$ ). See Fig. 7 .

For cases (i) and (ii), the hadronic cross section vanishes at $E=E_{C}$ accompanied by a very peculiar energy dependence. For small couplings, the spectral density as well as the elastic cross section peaks at around $E_{f}$ and the bound state, present in case (i), is a purely mesonic one. Then, with the increase of the coupling, the aforementioned bound state leaves the near-threshold region and acquires a large admixture of the quark component. In this strong-coupling regime the dynamics is defined by the presence of a single pole which corresponds to the virtual state. Due to the normalisation condition (21), the spectral density decreases with the increase of the coupling.

It is instructive to discuss, for example, Case (i) in some more detail. In particular, in Fig. 2, the movement of the poles in the $s$-plane is depicted on both first (left plot) and second (right plot) Riemann sheets. The arrows indicate how the poles start to move when $g_{f}$ is increased. Clearly this plot is equivalent to the pole movement in the $k$-plane depicted in the upper left panel of Fig. 1. As usual, for a narrow resonance ( $\operatorname{small} g_{f}$ ) only the pole in the lower half plane on the second sheet (see Fig. 2) influences the physics. Here this state is nearly a pure quark state. As $g_{f}$ gets larger the width of the state grows (and the mesonic admixture increases). At latest, when the real part of the pole position reaches the threshold (indicated in the figure as the perpendicular, dashed line), both poles are equally important. Notice that, in this regime, the interpretation of the imaginary part of the pole position as half of the width of the state is lost. Similar pole movements in the subthreshold regime were reported previously in Refs. 21, 22 .

In Cases (iii-a,b) one has $E_{C}<0$, so that the $t$-matrix zero does not manifest itself in the hadronic cross section defined for positive energies only. In the meantime, with $\left|E_{C}\right| \sim \Delta$, there are two bound states in the nearthreshold region. With the increase of the coupling, only one bound state survives in the near-threshold region, with $Z \rightarrow 0$. The spectral density is small for all values of the coupling, as two bound states saturate the normalisation condition (21).

Finally, in cases (iv-a) and (iv-b) and for small couplings, the bound state is almost purely of quark nature, and $E_{C}<0$. With the increase of the coupling, $E_{C}$ becomes positive and shows up in the hadronic cross section, while the bound state acquires a significant mesonic admixture. Further increase of the coupling forces the $t$ matrix zero to leave the near-threshold region, and the dynamics is defined by a single bound state, which is purely molecular, with $Z \rightarrow 0$.

So, for all cases considered, there is only one nearthreshold pole in the strong-coupling regime which corresponds to the scattering-length approximation for the mesonic $t$-matrix, independently of the underlying dynamics.

\section{Production reactions}

Unfortunately there is no experimental possibility to study elastic scattering of, say, charmed mesons, and our knowledge of the resonance properties comes from production experiments. In general there are two production mechanisms possible, namely a production of the resonance of interest via its hadronic component or via its quark component. If the production source can be considered as point-like, then the production amplitude of the meson pair $\left(M_{1} M_{2}\right)$ through the resonance from the former mechanism can be written as

$$
\mathcal{M}_{h}(E)=\mathcal{F}_{h}\left(1-\int d^{3} p \frac{t(\boldsymbol{p}, \boldsymbol{k}, E)}{p^{2} /(2 \mu)-E-i 0}\right)_{\mid k^{2} /(2 \mu)=E}
$$

where $\mathcal{F}_{h}$ is the initial state production amplitude from the point-like source, unity stands for the Born term, and the second term defines the final-state interaction - see the left panel of Fig. 8, For a point-like source and small energies, the amplitude (47) is

$$
\mathcal{M}_{h}(E)=\mathcal{F}_{h}(1-L(E) t(E))
$$


$L$ being the loop function describing propagation of the intermediate mesonic state,

$$
L(E)=4 \pi^{2} \mu\left(l_{0}+i k\right),
$$

with $l_{0} \sim \beta$, where $\beta$ is the range of the force. We may therefore write

$$
\mathcal{M}_{h}(E)=\mathcal{F}_{h} \frac{\left(E-E_{f}\right)\left(E_{f}-E_{C}\right)-\frac{1}{2} l_{0} g_{f}\left(E-E_{C}\right)}{\left(E-E_{f}\right)\left(E_{f}-E_{C}\right)+\frac{i}{2} k g_{f}\left(E-E_{C}\right)} .
$$

Thus, in $\mathcal{M}_{h}(E)$ the zero in the production amplitude is shifted with respect to the zero of the $t$-matrix. However, for the energies $E_{f} \simeq E_{C} \ll \beta$ this shift is small. The second term in the numerator of Eq. (50) dominates, and the production rate through the hadronic component alone is

$$
\begin{aligned}
\frac{d B r_{h}\left(M_{1} M_{2}\right)}{d E} & =\text { const } \times k|t(E)|^{2} \Theta(E) \\
& =\text { const } \times\left(\frac{g_{f}}{8 \pi^{2} \mu}\right)^{2} \frac{k}{\left|\mathcal{D}_{F}\right|^{2}},
\end{aligned}
$$

where the denominator $\mathcal{D}_{F}$ is given by Eq. (31). Thus one might expect that all said above about the physical content of a possible zero in $t$-matrix in the nearthreshold regime translates one-to-one also to production reactions. However, this is not correct for there is, in addition to the hadronic production, also the production via the quark component possible. This piece can be expressed via the spectral density. Indeed, as seen from the right panel Fig. 8, the corresponding production amplitude is given by:

$$
\mathcal{M}_{q}=-\mathcal{F}_{q} G_{q 0}(E) t_{q h}(\boldsymbol{k}, E), \quad \frac{k^{2}}{2 \mu}=E,
$$

where $\mathcal{F}_{q}$ is the production amplitude for the bare quark state by the point-like source, $G_{q 0}(E)=1 /\left(E_{0}-E\right)$ is the quark state free Green's function, and $t_{q h}(\boldsymbol{k}, E)$ is the $t$-matrix element responsible for the quark-meson transition. From the corresponding Lippmann-Schwinger equation,

$$
t_{q h}(\boldsymbol{k}, E)=f(\boldsymbol{k})-\int d^{3} p \frac{f(\boldsymbol{p}) t(\boldsymbol{p}, \boldsymbol{k}, E)}{p^{2} /(2 \mu)-E-i 0},
$$

and with the help of Eqs. (18), (19), the latter can be found in the form:

$$
t_{q h}(\boldsymbol{k}, E)=\int d^{3} p \chi_{k}(\boldsymbol{p}) f(\boldsymbol{p})=\left(E-E_{0}\right) c_{k}(E) .
$$

Thus, the near-threshold production rate via the quark component of the wave function is simply

$$
\frac{d B r_{q}\left(M_{1} M_{2}\right)}{d E}=\text { const } \times w(E),
$$

where $w(E)$ is defined in Eq. (20). Then, using definition (20) and the explicit form of the coefficient $c_{k}(E)$, which follows from Eq. (18),

$$
c_{k}(E)=\sqrt{\frac{g_{f}}{8 \pi^{2} \mu}} \frac{E_{f}-E_{C}}{E-E_{C}} \frac{1}{\mathcal{D}_{F}},
$$

one finds:

$$
w(E)=\frac{1}{2 \pi} \frac{k g_{f}}{\left|\mathcal{D}_{F}\right|^{2}} \frac{\left(E_{f}-E_{C}\right)^{2}}{\left(E-E_{C}\right)^{2}} .
$$

Obviously, this implies that $d B r_{q} / d E$ does not employ a zero.

In reality one expects both mentioned mechanisms to contribute to the production of the physical resonance, with a relative importance depending on the production mechanism. Therefore, we may write for the full production rate:

$$
\frac{d B r\left(M_{1} M_{2}\right)}{d E}=\text { const } \times k \frac{\left|E-E_{C}+r\left(E_{f}-E_{C}\right)\right|^{2}}{\left|\mathcal{D}_{F}\right|^{2}\left(E-E_{C}\right)^{2}},
$$

where $r$ is a real number containing, among other contributions, the ratio of the production rates via the quark state and the hadronic state. A priori no estimate of $r$ is possible thus, even if there were a system that shows a zero in the scattering amplitude near threshold and allowing for all the conclusions of the previous section, this zero might well be shielded in the production reaction. On the other hand, if $E_{c} \gg \Delta$ it requires a delicate fine tuning of the parameter $r$ to produce a zero in the amplitude of Eq. (58). Thus, if there is a zero observed in a production amplitude, it at least suggests the presence of both quark states as well as a hadronic molecule.

Note that the zero in the lineshape of the $X(3872)$ predicted to occur in a $B$-decay amplitude reported in Ref. 23, 24] is of a different kind, for it originates as a coupled-channel effect of two nearby continuum channels. In this work, on the other hand, the effects in the presence of only a single continuum channel were discussed.

\section{Implications for lattice QCD}

From the previous discussions it should be clear that the presence of a zero in the scattering amplitude contains important information about the system studied. However, this information is no longer visible in the production amplitude, which is the quantity accessible experimentally.

Fortunately scattering observables, in particular phase shifts, are accessible in lattice QCD from a study of volume dependencies of the energy spectra 25]. In simulations of full QCD both quark states as well as molecular states are present and will influence all correlators, as long as some overlap exists with the interpolating fields used. Thus, the results of those simulations need to be interpreted in the same way as experimental results call for an interpretation, if one wants to gain some insight into the dynamical mechanisms that lead to the structure formation. One method pushed recently is to work with a large basis of interpolating fields and to use the resulting eigenvectors of the correlator matrices to interpret the findings [26,27]. In Ref. 22 the scattering length is shown to be an important quantity also to extract information about the nature of states from lattice studies. The discussion above provides one with an additional approach: in order to understand 
the interplay of quark and meson degrees of freedom one may investigate the near-threshold phase shifts. If a zero in the $t$-matrix, corresponding to a zero in the phase shifts, occurs, it immediately signals the presence of both quark poles as well as potential scattering poles with a relatively weak coupling as described in Sec. 4.

To illustrate this point we discuss briefly the phase shift (its cotangent) for Case (i) introduced above,

$$
k \cot \delta=-\frac{\gamma_{V}\left(E-E_{f}\right)}{E-E_{f}+\frac{1}{2} g_{f} \gamma_{V}} .
$$

This expression employs a pole at the position of the $t$ matrix zero, as shown in Fig. 9. We expect that such a structure could be extracted from studies within lattice QCD.

\section{Conclusions}

In this paper we have presented a dynamical scheme in which a near-threshold zero in the mesonic $t$-matrix appears as a consequence of the interplay between quark states and a nonperturbatively interacting hadron-hadron continuum. The appearance of such a zero invalidates the effective-range expansion and corresponds to three nearthreshold poles of the $t$-matrix. The effect on hadronic observables in elastic scattering is proved to be drastic.

However, a near-threshold $t$-matrix zero could exist only if several requirements are met. First, one needs the direct interaction in the mesonic channel to be strong enough to support a bound or virtual state. Second, a nearby bare quark state should exist, with a weak coupling to the mesonic channel. While, in principle, such a situation cannot be excluded, it is highly accidental.

Without such special arrangements the effective-range formulae are valid, and the conclusions of Refs. 11, 12, 13, 14, 15, 16, hold true: a large and negative effective range corresponds to a compact quark state, while a small effective range means that the state is composite. In the former case there are two near-threshold poles in the $t$ matrix, while in the latter case there is only one pole.

In the two-pole case one can definitely state that the resonance is generated by an $s$-channel diagram. The coupling of this quark state to the mesonic continuum is not large. In the one-pole situation no model-independent insight into the underlying dynamics is possible and one can only indicate that the resonance is generated dynamically but, in the language of meson exchange, the binding potential could emerge from either $s$-channel of $t$-channel exchanges - or a mixture of both. It seems not possible to decide between these scenarios model independently. The three pole scenario, on the other hand, calls for the presence of both $s$-channel interactions as well as potential scattering — traditionally identified with $t$ channel exchanges. This situation can be identified easily by the break-down of the effective-range expansion. Although scattering experiments are not possible for most unstable particles, an alternative access to scattering observables could be provided by studies of lattice QCD.
We also discussed the implications for production reactions. Unfortunately it turns out that for the lineshapes of production reactions no statement is possible model independently based solely on information from the scattering amplitudes.

The work was supported in parts by funds provided from the Helmholtz Association (grants VH-NG-222, VH-VI-231), by the DFG (grants SFB/TR 16 and 436 RUS 113/991/0-1), by the EU HadronPhysics2 project, by the RFFI (grants RFFI-0902-91342-NNIOa and RFFI-09-02-00629a), and by the Presidential programme for support of the leading scientific schools (grants NSh-4961.2008.2 and NSh-4568.2008.2). The work of V.B., Yu.K., A.K., and A.N. was supported by the State Corporation of Russian Federation "Rosatom". A. N. would like to acknowledge the support of the non-profit "Dynasty" foundation and ICFPM and of the grant PTDC/FIS/70843/2006Fisica.

\section{References}

1. E. S. Swanson, Phys. Rept. 429 (2006) 243.

2. E. Klempt and A. Zaitsev, Phys. Rept. 454 (2007) 1.

3. M. B. Voloshin, Prog. Part. Nucl. Phys. 61 (2008) 455.

4. S. Godfrey and S. L. Olsen, Ann. Rev. Nucl. Part. Sci. 58 (2008) 51.

5. E. Braaten, arXiv:0808.2948 [hep-ph].

6. S.-K. Choi et al. [Belle Collaboration], Phys. Rev. Lett. 91 (2003) 262001.

7. B. Aubert et al. [BABAR Collaboration], Phys. Rev. Lett. 95 (2005) 142001.

8. B. Aubert et al. [BABAR Collaboration], Phys. Rev. Lett. 98 (2007) 212001.

9. S.-K. Choi et al. [Belle Collaboration], Phys. Rev. Lett. 100 (2008) 142001.

10. R. Mizuk et al. [Belle Collaboration], Phys. Rev. D $\mathbf{7 8}$ (2008) 072004.

11. S. Weinberg, Phys. Rev. 130 (1963) 776.

12. S. Weinberg, Phys. Rev. 131 (1963) 440.

13. S. Weinberg, Phys. Rev. 137 (1965) B672.

14. V. Baru et al., Phys. Lett. B 586 (2004) 53.

15. V. Baru et al., Eur. Phys. Journ. A 23 (2005) 523.

16. D. Morgan, Nucl. Phys. A 543 (1992) 632.

17. L. N. Bogdanova, G. M. Hale, and V. E. Markushin, Phys. Rev. C 44 (1991) 1289.

18. M. Döring, C. Hanhart, F. Huang, S. Krewald and U. G. Meißner, Phys. Lett. B 681 (2009) 26.

19. M. Döring, C. Hanhart, F. Huang, S. Krewald and U. G. Meißner, Nucl. Phys. A 829 (2009) 170.

20. S. Flatté, Phys. Lett. B 63 (1976) 224.

21. C. Hanhart, J. R. Pelaez, and G. Rios, Phys. Rev. Lett. 100 (2008) 152001.

22. F. K. Guo, C. Hanhart, and U. G. Meißner, Eur. Phys. J. A 40 (2009) 171.

23. E. Braaten and M. Lu, Phys. Rev. D 77 (2008) 014029.

24. M. B. Voloshin, Phys. Rev. D 76 (2007) 014007.

25. M. Lüscher, Commun. Math. Phys. 105 (1986) 153.

26. J. J. Dudek, R. G. Edwards, M. J. Peardon, D. G. Richards, and C. E. Thomas, Phys. Rev. Lett. 103 (2009) 262001.

27. J. J. Dudek, R. G. Edwards, M. Mathur, and D. G. Richards, Phys. Rev. D 77 (2008) 034501. 

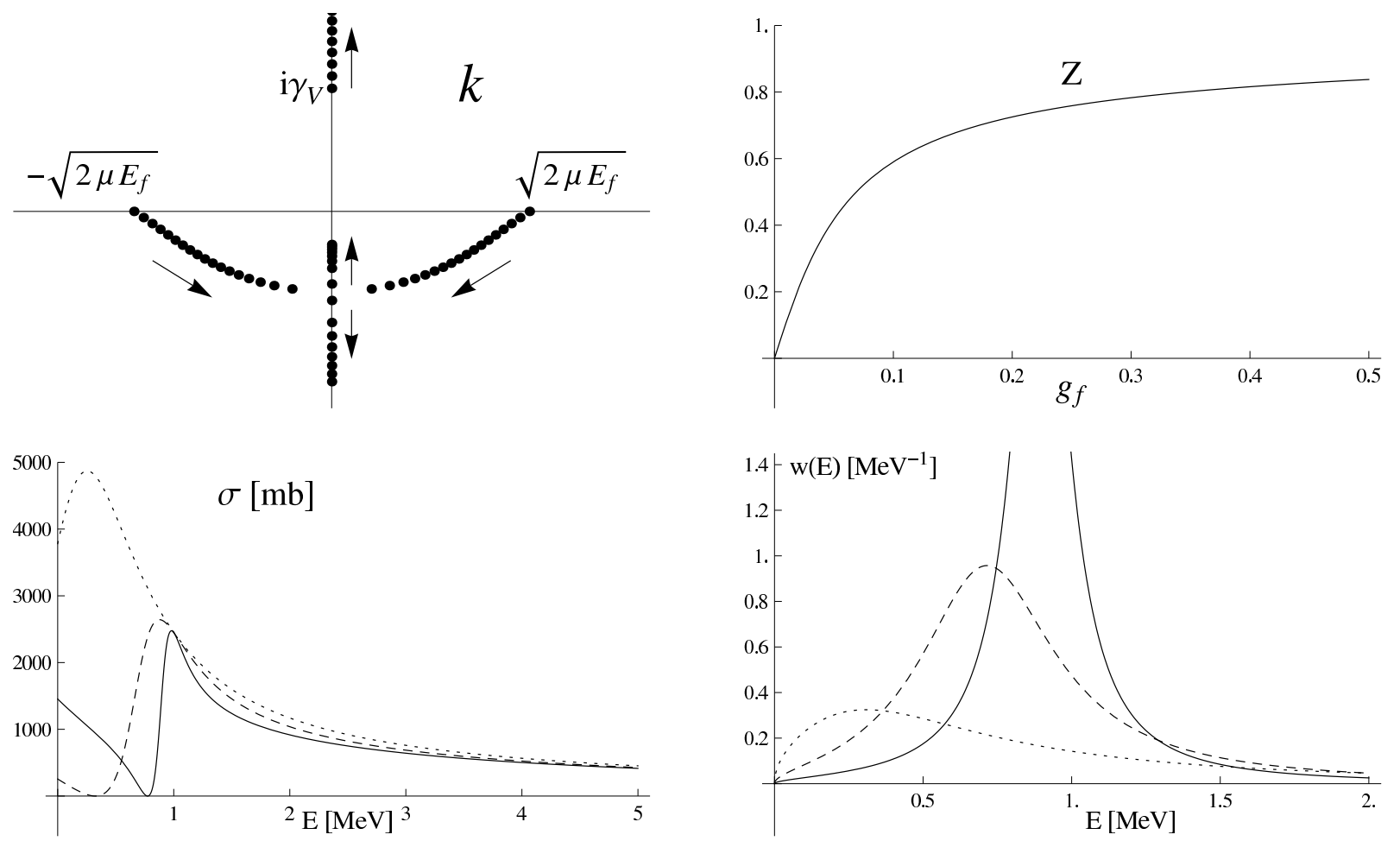

Fig. 1. Case (i). Upper panel: the pole structure in the $k$-plane (left plot) and the $Z$-factor - see Eq. (36) (right plot) versus the coupling constant $g_{f}$. Lower panel: the elastic scattering cross section (left plot) and the spectral density (right plot) versus the energy for $g_{f}=0.01$ (solid line), $g_{f}=0.03$ (dashed line), and $g_{f}=0.1$ (dotted line).
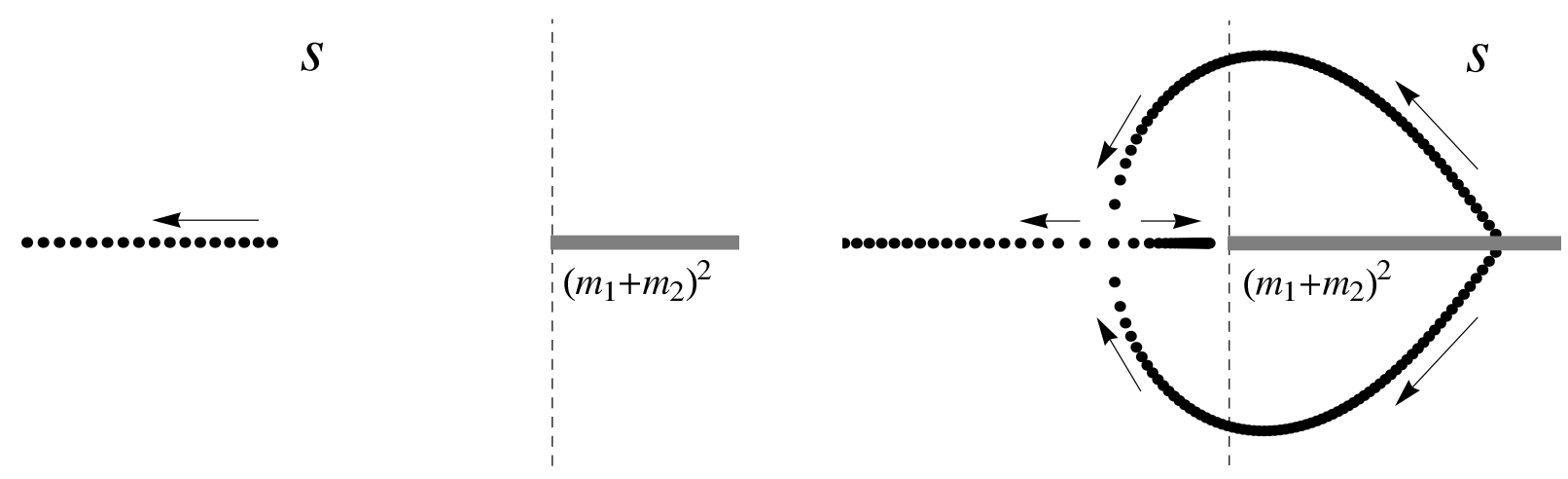

Fig. 2. The poles motion in the s-plane for Case (i) at the first Riemann sheet (left plot) and on the second Riemann sheet (right plot). The unitarity cut is depicted in grey. 

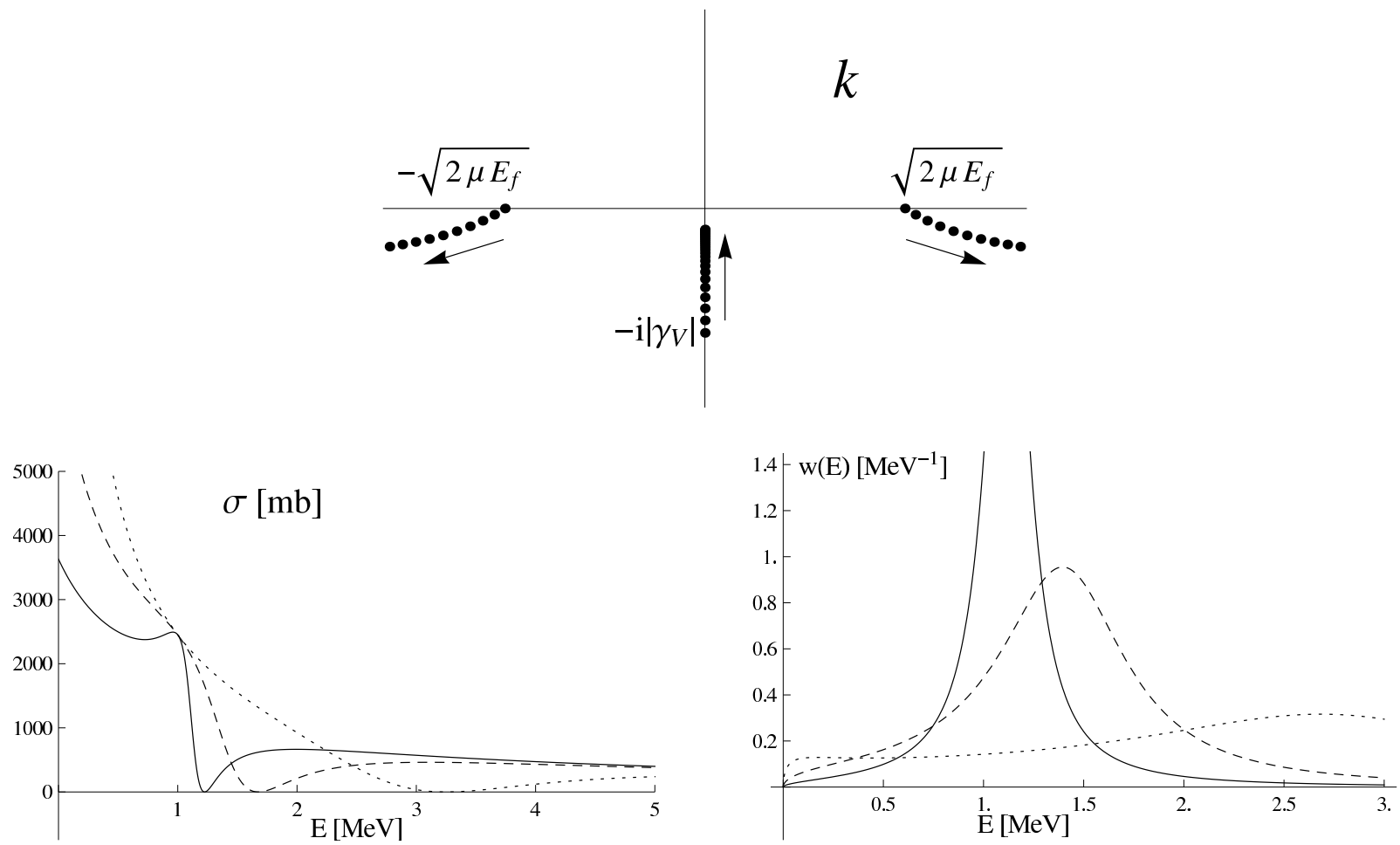

Fig. 3. Case (ii). Upper panel: the pole structure in the $k$-plane. Lower panel: the elastic scattering cross section (left plot) and the spectral density (right plot) versus the energy for $g_{f}=0.01$ (solid line), $g_{f}=0.03$ (dashed line), and $g_{f}=0.1$ (dotted line).
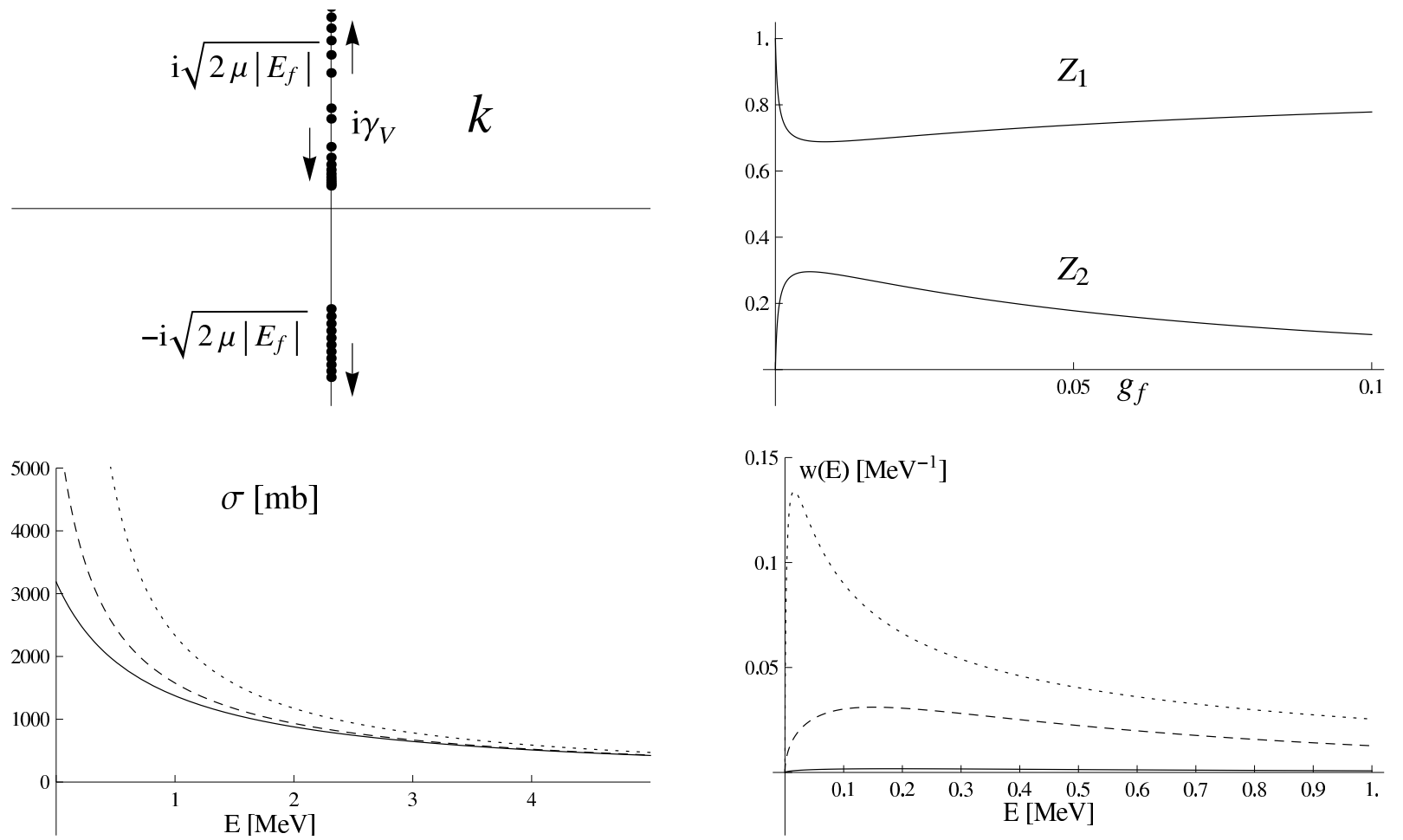

Fig. 4. Case (iii-a). Upper panel: the pole structure in the $k$-plane (left plot) and the $Z$-factors - see Eq. (36) (right plot) versus the coupling constant $g_{f}$. Lower panel: the elastic scattering cross section (left plot) and the spectral density (right plot) versus the energy for $g_{f}=0.001$ (solid line), $g_{f}=0.02$ (dashed line), and $g_{f}=0.3$ (dotted line). 

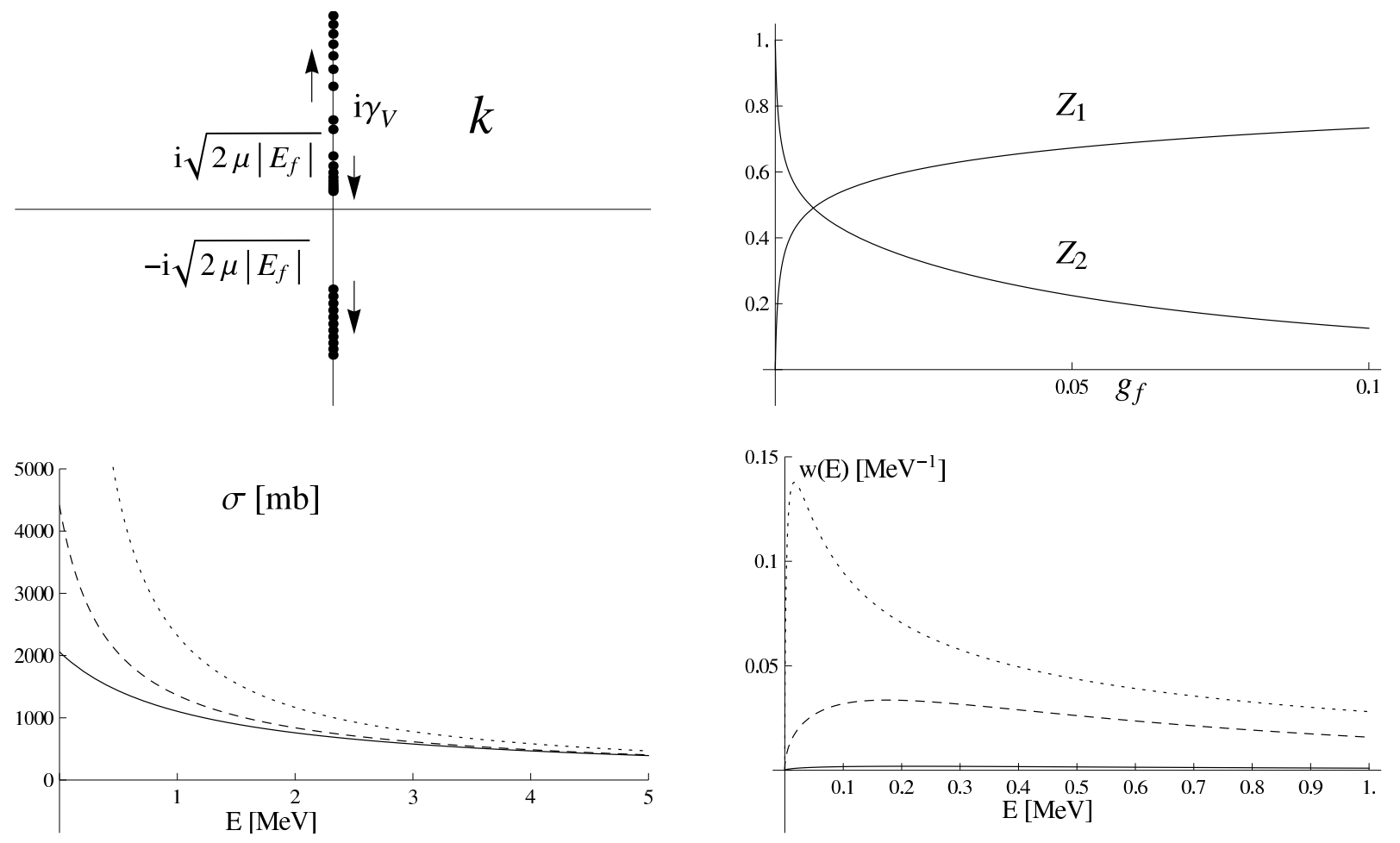

Fig. 5. Case (iii-b). Upper panel: the pole structure in the $k$-plane (left plot) and the $Z$-factors (right plot) versus the coupling constant $g_{f}$. Lower panel: the elastic scattering cross section (left plot) and the spectral density (right plot) versus the energy for $g_{f}=0.001$ (solid line), $g_{f}=0.02$ (dashed line), and $g_{f}=0.3$ (dotted line).
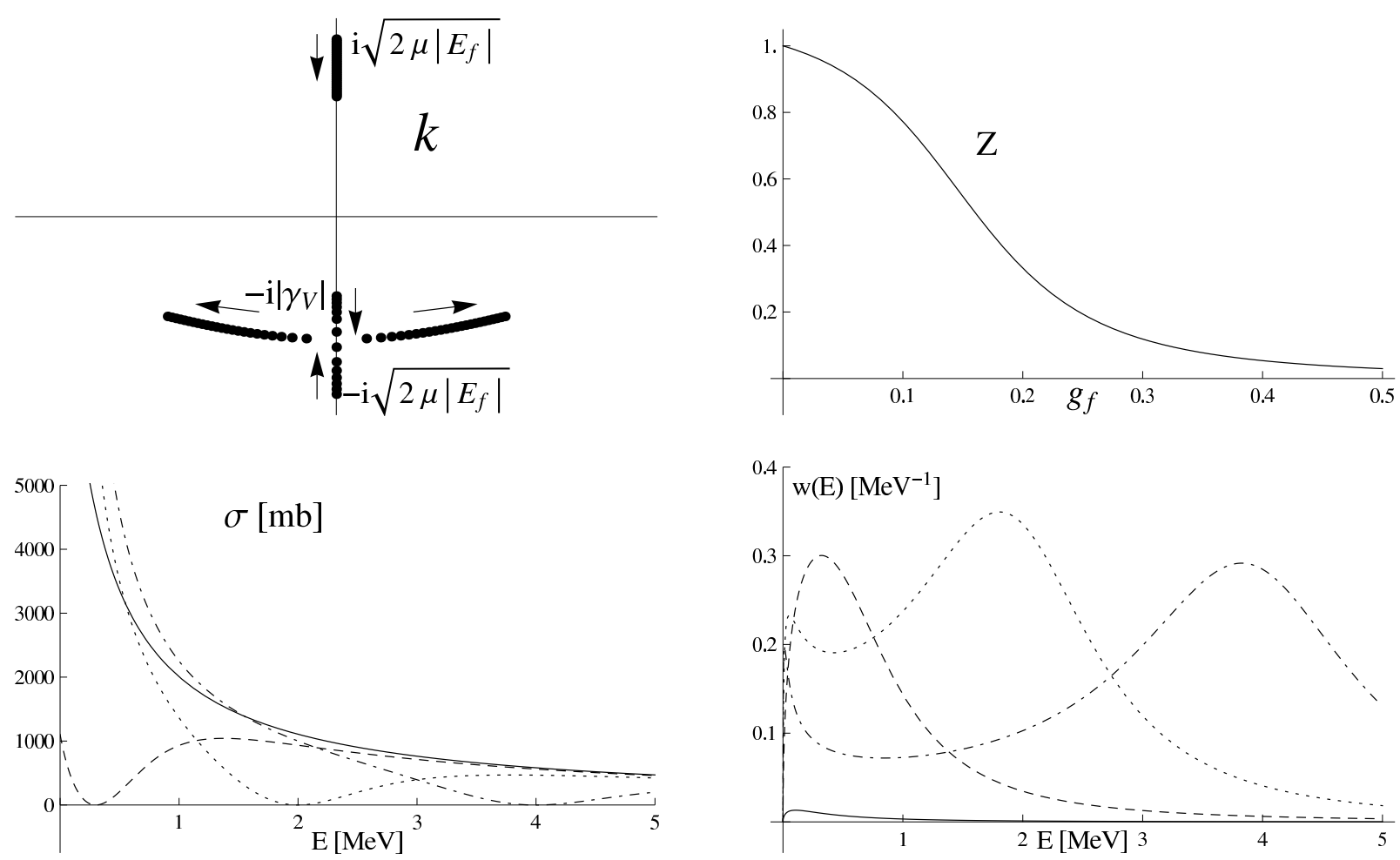

Fig. 6. Case (iv-a). Upper panel: the pole structure in the $k$-plane (left plot) and the $Z$-factor - see Eq. (36) (right plot) versus the coupling constant $g_{f}$. Lower panel: the elastic scattering cross section (left plot) and the spectral density (right plot) versus the energy for $g_{f}=0.01$ (solid line), $g_{f}=0.13$ (dashed line), $g_{f}=0.3$ (dotted line), and $g=0.5$ (dash-dotted line). 

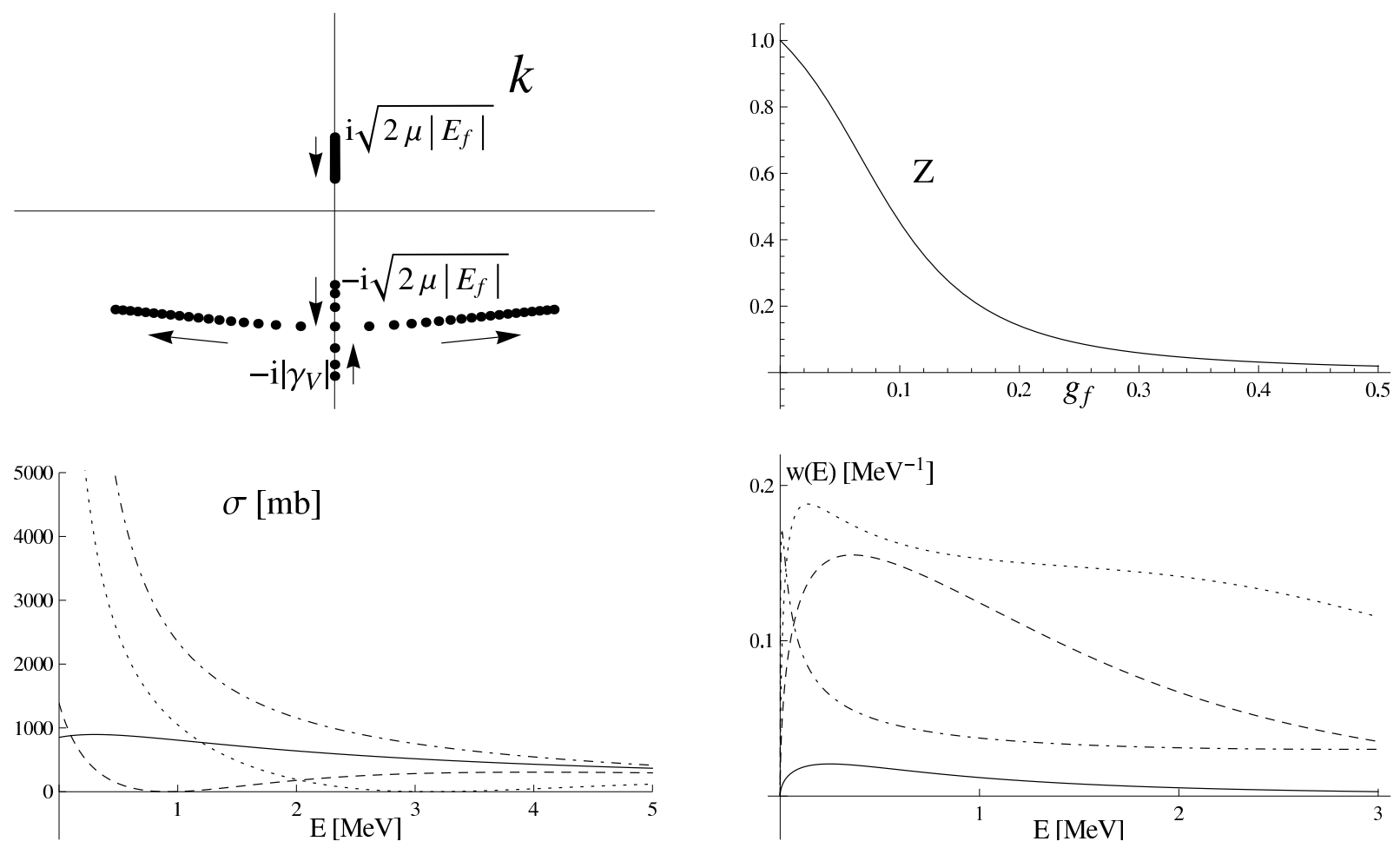

Fig. 7. Case (iv-b). Upper panel: the pole structure in the $k$-plane (left plot) and the $Z$-factor - see Eq. (36) (right plot) versus the coupling constant $g_{f}$. Lower panel: the elastic scattering cross section (left plot) and the spectral density (right plot) versus the energy for $g_{f}=0.01$ (solid line), $g_{f}=0.07$ (dashed line), $g_{f}=0.15$ (dotted line), and $g=0.5$ (dash-dotted line).
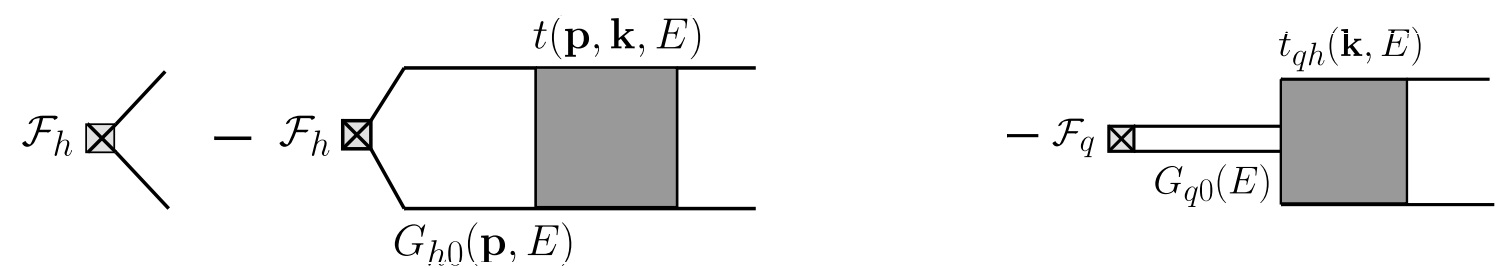

Fig. 8. Diagrams for the two meson production via the hadronic component (left plot) and via the quark component (right plot).

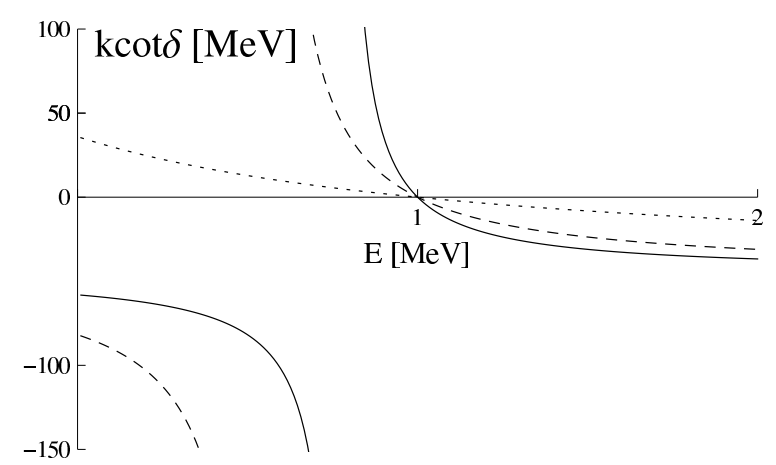

Fig. 9. The phase shift for Case (i) for $g_{f}=0.01$ (solid line), $g_{f}=0.02$ (dashed line), and $g_{f}=0.1$ (dotted line). 